\title{
A Brief Introduction
}

This book contains 296 solved exercises on the theory of linear models. The exercises are taken from the author's graduate-level textbook, Linear Model Theory: With Examples and Exercises, which was published by Springer in 2020. The exercises themselves have been restated, when necessary and feasible, to make them as comprehensible as possible independently of the textbook, but the solutions refer liberally to theorems and other results therein. They are arranged in chapters, the numbers and titles of which are identical to those of the chapters in the textbook that have exercises.

Some of the exercises and solutions are short, while others have multiple parts and are quite lengthy. Some are proofs of theorems presented but not proved in the aforementioned textbook, but most are specializations of said theorems and other general results to specific linear models. In this respect they are quite similar to the textbook's examples. A few of the exercises require the use of a computer, but none involve the analysis of actual data.

The author is not aware of any other published set of solved exercises for a graduate-level course on the theory of linear models. It is hoped that students and instructors alike, possibly even those not using Linear Model Theory: With Examples and Exercises for their course, will find these exercises and solutions useful. 